\title{
PADRONIZAÇÃO DAS TÉCNICAS OPERATÓRIAS DE AUTONOMIZAÇÃO NO RETALHO MÚSCULOCUTÂNEO TRANSVERSO DO MÚSCULO RETO DO ABDOME MONOPEDICULADO. ESTUDO EXPERIMENTAL EM RATAS ${ }^{1}$
}

Rui Lopes Filho ${ }^{2}$

Alcino Lázaro da Silva ${ }^{3}$

\begin{abstract}
Lopes Filho R, Silva AL. Padronização experimental das técnicas operatórias de autonomização no retalho musculocutâneo transverso do músculo reto do abdome monopediculado: estudo experimental em ratas. Acta Cir Bras [serial online] 2002 Jul-Ago;17(4). Disponível em URL: http://www.scielo.br/acb.
\end{abstract}

RESUMO - Objetivo: Padronizar as técnicas operatórias de autonomização aplicadas previamente ao descolamento e elevação do músculo reto do abdome (Retramcur) com sutura no próprio leito e estabelecer qual delas é a mais efetiva na redução da necrose do retalho. Métodos: Oitenta ratas Wistar, pesando entre 250gramas a 300gramas foram distribuídas aleatoriamente em oito grupos de dez ratas $(\mathrm{n}=10)$. O grupo A foi considerado o grupo controle, no qual as ratas foram submetidas ao descolamento e elevação do Retramcur, com sutura no próprio leito. Os animais dos grupos B, C, D, E, F, G e H foram submetidos a diferentes técnicas operatórias de autonomização previamente ao descolamento e elevação do Retramcur, com sutura no próprio leito. As medidas das áreas de necrose do Retramcur foram realizadas no quinto dia pós-operatório, utilizando-se um molde retirado de uma folha de transparência, caneta de ponta fina Pilot ${ }^{\circledR}$ e folha milimetrada padronizada, papéis técnicos Foroni â, na qual foi realizada a medida da extensão da área de necrose de cada retalho em porcentagem. Resultados: Para análise estatística dos resultados foi aplicado o teste para diferenças significativas mínimas ou LSD (Least Significante Difference). O grupo A (controle) apresentou, em média, porcentual de área de necrose estatisticamente superior em relação aos demais grupos. A técnica operatória utilizada nos animais do grupo $\mathrm{C}$ apresentou, em média, porcentual de área de necrose estatisticamente inferior a dos grupos H, B, F e G e semelhante a dos grupos D e E. Conclusão: Os resultados encontrados permitem concluir que a técnica operatória de autonomização mais efetiva na redução da área de necrose do Retramcur é a que seccionou ambos os músculos retos do abdome e cauterizou ambos os vasos epigástricos superiores profundos, utilizada nos animais do grupo C.

DESCRITORES - Autonomização. Retalhos cirúrgicos. Necrose. Reto do abdome. Ratos.

\section{INTRODUÇÃO}

O retalho musculocutâneo transverso do músculo reto do abdome (Retramcur) é constituído de um segmento horizontal de pele, tela subcutânea e músculo, descolado do abdome inferior, permanecendo nutrido pelos vasos epigástricos superiores profundos, a fim de transpô-lo para o tórax 1,2.
Os princípios básicos para a utilização do Retramcur (TRAM - flap) foram descritos por HARTRAMPF et al. (1982), consagrando-se como uma das melhores alternativas de reconstrução das mamas com tecidos autógenos e sem a necessidade do uso dos implantes mamários, após mastectomias.

O grande problema na utilização do referido retalho com apenas um músculo reto do abdome e que motivou

1. Trabalho realizado no Curso de Pós-Graduação em Cirurgia da Faculdade de Medicina - Universidade Federal de Minas Gerais (UFMG).

2. Membro Especialista da Sociedade Brasileira de Cirurgia Plástica.

3. Professor Titular, Doutor de Cirurgia do Aparelho Digestivo da Faculdade de Medicina - UFMG। 
o estudo, foi a presença de necrose de pele no retalho, particularmente nas pacientes que apresentam os fatores de risco descritos por HARTRAMPF (1988) 3,4,5,6,7.

Os fatores de risco para a reconstrução mamária com o Retramcur são a obesidade, as vasculopatias associadas ao tabagismo e ao diabetes, os problemas psicológicos, as cicatrizes no abdome, as doenças pulmonares crônicas, as doenças cardiovasculares graves e a inexperiência do cirurgião, que aumenta a amplitude do problema 6,7 .

As técnicas operatórias de autonomização determinam uma isquemia tecidual programada e intencional nos retalhos, fazendo com que eles passem a sobreviver com um fluxo sanguíneo menor que o normal, provocando um estímulo ao aumento da vascularização interna, através de procedimentos operatórios prévios, que proporcionam a diminuição das áreas de necrose destes retalhos 8,9 .

$\mathrm{Na}$ tentativa de reduzir a necrose do Retramcur (TRAM flap), encontramos estudos na literatura, que utilizaram técnicas operatórias de autonomização em animais 10,11,12,13,14.

ÖZGENTAS et al. (1994) estabeleceram um modelo experimental de Retramcur em ratos, com a vascularização proveniente dos vasos epigástricos inferiores profundos, que foi o pedículo vascular não dominante.

Os objetivos desta pesquisa são padronizar as técnicas operatórias de autonomização utilizadas no Retramcur monopediculado, e estabelecer qual delas é a mais eficaz na redução da área de necrose dos retalhos previamente autonomizados.

\section{MÉTODOS}

Utilizou-se 80 ratas Wistar pesando entre 250 gramas a 300gramas, alimentadas com ração (Labina, ração para ratos, PURINA Campinas, São Paulo) e água à vontade. A guarda e os procedimentos técnicos foram realizados no laboratório experimental do Curso de Pósgraduação em Cirurgia da Faculdade de Medicina, UFMG.

A anestesia utilizada para as operações e obtenção das medidas das áreas de necrose foi IM, utilizando-se o Cloridrato de Cetamina (Ketalar $®$ ), na dosagem de $6 \mathrm{mg}$ a $10 \mathrm{mg} / \mathrm{kg}$ de peso associado a $0,1 \mathrm{ml} / \mathrm{kg}$ de Cloridrato de 2-(2,6 Xilidino)-5,6-Dihidro-4H-1,3Tiazina(Rompunâ).

O Retramcur proposto para o estudo constituiuse de um segmento retangular e transverso de pele fixa ao músculo reto do abdome esquerdo, medindo $4,2 \mathrm{~cm}$ por $2,1 \mathrm{~cm}$, sendo desenhado no abdome superior por meio de um molde retirado de uma folha de radiografia, sendo o limite superior do retalho representado pela linha horizontal que passa pelo apêndice xifóide.
O segmento de pele do retalho foi dividido em áreas 1,2 e 3 . A área 1 localizou-se sobre o músculo reto do abdome esquerdo, a área 3 no segmento de pele contralateral ao pedículo vascular do retalho e a área 2 entre as áreas 1 e 3 (Figura 1).

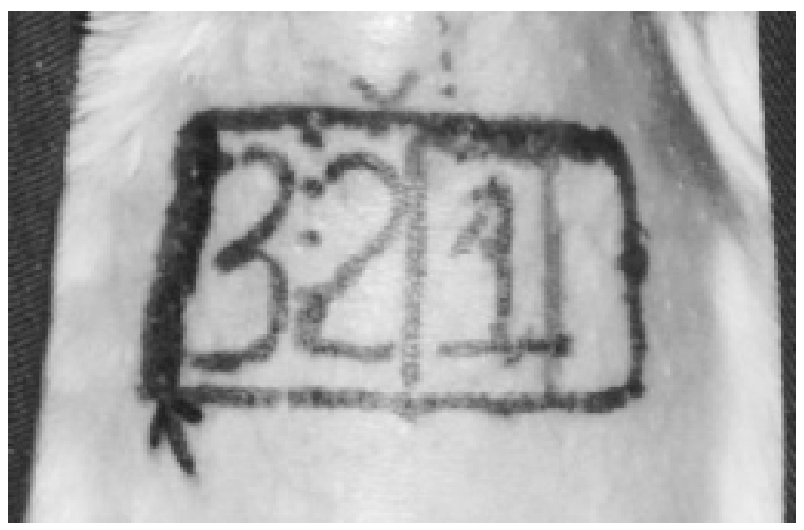

Figura 1 - Desenho das áreas 1, 2 e 3 do Retramcur. Projeção do músculo reto do abdome na área 1 . A seta indica o segmento de pele contralateral ao pedículo vascular do retalho e mais susceptível à necrose.

Os animais foram divididos em oito grupos identificados pelas letras A, B, C, D, E, F, G e H, compostos de dez ratas $(n=10)$, de acordo com a técnica operatória de autonomização utilizada, previamente ao descolamento e elevação do Retramcur, sendo o período de autonomização de sete dias. As técnicas operatórias de autonomização realizadas nos animais dos grupos B, C, D, E, F, G e H, encontram-se em pormenores no Quadro 1.

O grupo A foi o grupo controle do estudo, no qual não foi realizada nenhuma técnica operatória de autonomização, previamente a operação do Retramcur, que foi descolado e rebatido até o abdome inferior, seguido de sutura no próprio leito com mononáilon 4-0 (Figura 2).

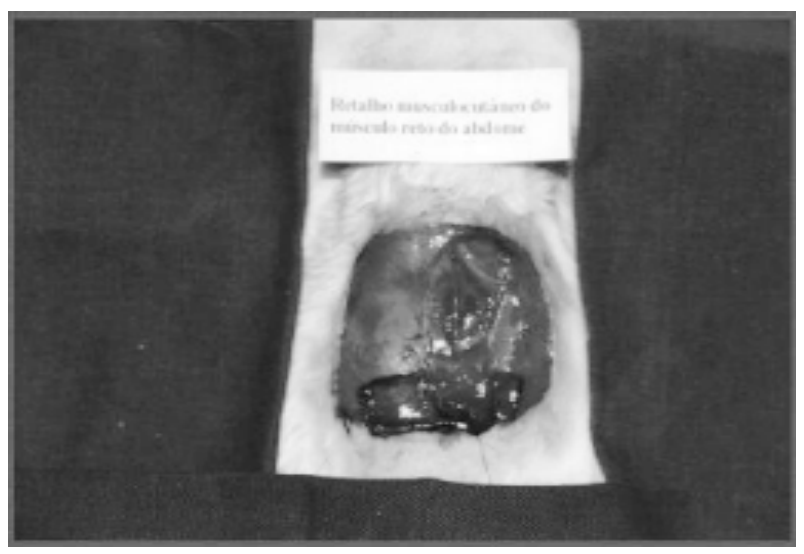

Figura 2 - Vê-se Retramcur descolado e rebatido até o abdome inferior. 
Quadro 1 - Técnica operatória de autonomização utilizada em cada grupo.

\begin{tabular}{|c|l|}
\hline GRUPO & \multicolumn{1}{c|}{ AUTONOMIZAÇÃO } \\
\hline B & $\begin{array}{l}\text { Cranial de pele, tela subcutânea, músculo reto do abdome esquerdo e vasos epigástricos } \\
\text { superiores profundos }\end{array}$ \\
\hline C & $\begin{array}{l}\text { Bilateral cranial de pele, tela subcutânea, músculos retos do abdome e vasos epigástricos } \\
\text { superiores profundos }\end{array}$ \\
\hline D & Incisão nos limites do retalho sem descolamento \\
\hline E & Associando-se as autonomizações B e D \\
\hline F & Associando-se as autonomizações C e D \\
\hline G & $\begin{array}{l}\text { Cranial de pele, tela subcutâneas, músculo reto do abdome esquerdo, vasos epigástricos } \\
\text { superiores profundos, associada a autonomização pele, tela subcutânea, cauterização de } \\
\text { perfurante musculocutânea, com introdução de lâmina de silicone na área 2 }\end{array}$ \\
\hline H & $\begin{array}{l}\text { Incisão nos limites do retalho sem descolamento, associada ao descolamento, cauterização } \\
\text { de perfurante musculocutânea e intrudução de lâmina de silicone na área 2 }\end{array}$ \\
\hline
\end{tabular}

A segunda operação realizada nos retalhos previamente autonomizados dos animais dos grupos B, C, D, E, F, G e H, foi semelhante a do grupo A (controle). O período de autonomização foi de sete dias.

As medidas das áreas de necrose dos retalhos foram realizadas no quinto dia pós-operatório, utilizandose um molde retirado de uma folha de transparência com as medidas do retalho em cicatrização, que foi colocado sobre o abdome dos animais, no qual foi realizado o desenho da área de necrose com uma caneta Pilot $\hat{a}$ para retro projetor de ponta fina.

O molde foi colocado sobre uma folha milimetrada padronizada, papéis técnicos Foroniâ, na qual foi realizada as medidas das áreas de necrose, sendo obtida a porcentagem de área de necrose, em relação à área total do retalho em cicatrização.

\section{RESULTADOS}

\section{Alterações Macroscópicas}

As alterações macroscópicas foram representadas pela necrose de pele nos retalhos, que apresentou distribuição e contorno variável em cada grupo, iniciando-se com 48 horas, estando definida no quarto dia pós-operatório (Figura 3).

Os retalhos dos animais do grupo A (controle) apresentaram necrose nas áreas 2 e 3, parte lateral da área 1 , não sendo verificada necrose sobre o músculo reto do abdome.

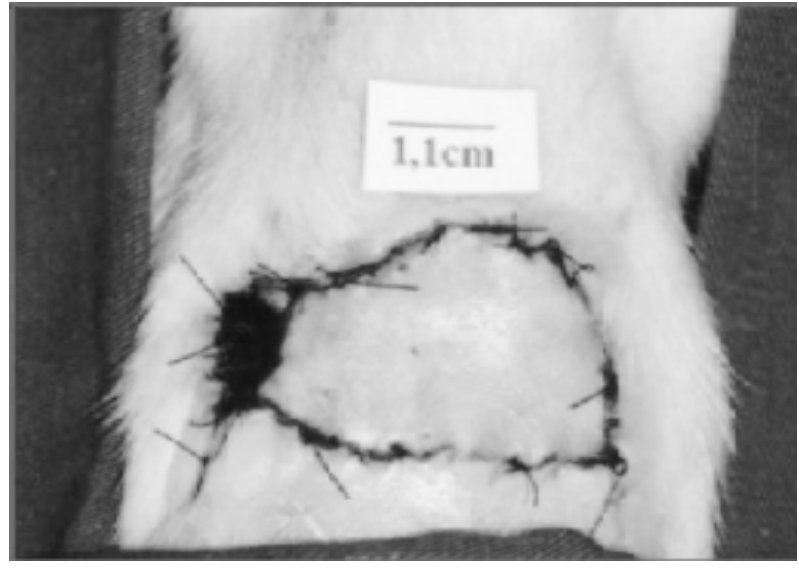

Figura 3 - Vê-se área de necrose no Retramcur.

Os retalhos dos animais do grupo $\mathrm{B}$ apresentaram necrose na área 3 e na parte lateral da área 2, não sendo verificada necrose na área $1 \mathrm{e}$ em parte da área 2.

Os retalhos dos animais do grupo $\mathrm{C}$ apresentaram necrose na parte lateral da área 3 , não sendo verificada necrose nas áreas 1 e 2 . Em seis ratas do grupo $\mathrm{C}$ constatou-se sobrevida total dos retalhos.

Os retalhos dos animais do grupo D apresentaram necrose na área 3 e na parte lateral da área 2, não sendo verificada necrose na área 1 . Em três ratas do grupo $D$ constatou-se sobrevida total dos retalhos.

Os retalhos dos animais do grupo $\mathrm{E}$ apresentaram necrose na área 3 e parte da área 2 , não sendo verificada necrose na área 1. Em seis ratas do grupo E constatouse sobrevida total dos retalhos.

Os retalhos dos animais do grupo $\mathrm{F}$ apresentaram necrose em toda área 3 e em parte da área 2, não sendo 
verificada necrose na área 1. Em três ratas do grupo D constatou-se sobrevida total dos retalhos.

Os retalhos dos animais do grupo E apresentaram necrose na área 3 e parte da área 2, não sendo verificada necrose na área 1. Em seis ratas do grupo E constatouse sobrevida total dos retalhos.

Os retalhos dos animais do grupo $\mathrm{F}$ apresentaram necrose em toda área 3 e em parte da área 2, não sendo verificada necrose na área 1 . Em três ratas do grupo $\mathrm{F}$ constatou-se sobrevida total dos retalhos.

Os retalhos dos animais do grupo $\mathrm{G}$ apresentaram necrose em toda área 3 e parte da área 2, não sendo verificada necrose na área 1 . Uma rata do grupo $\mathrm{G}$ apresentou sobrevida total do retalho. Os retalhos dos animais do grupo $\mathrm{H}$ apresentaram necrose em toda área 3 e parte da área 2, não sendo verificada necrose na área 1. Uma rata do grupo $\mathrm{H}$ apresentou sobrevida total do retalho.

\section{Análise Estatística}

De acordo com os resultados apresentados na Tabela 1 e no Gráfico 1, o grupo A (controle) apresentou, em média, porcentual de área de necrose estatisticamente superior, quando comparado com os demais grupos, nos quais foram realizadas técnicas operatórias de autonomização $(\mathrm{p}<0,01)$.

A técnica operatória utilizada no grupo C apresentou, em média, porcentual de área de necrose estatisticamente inferior a dos grupos H, B, F e G $(\mathrm{p}<0,01)$.

As técnicas operatórias utilizadas nos grupos D e E apresentaram, em média, porcentual de área de necrose estatisticamente semelhante em relação ao grupo $\mathrm{C}(\mathrm{p}>0,01)$.

Tabela 1 - Caracterização dos retalhos avaliados em relação ao porcentual de área de necrose nos grupos A, B, C, D, E, F, G e H.

\begin{tabular}{|c|c|c|c|c|c|c|}
\hline \multirow[b]{2}{*}{ Grupo } & \multicolumn{5}{|c|}{ Medidas descritivas } & \multirow[b]{2}{*}{ p/ conclusão } \\
\hline & Mínimo & Máximo & Média & DP & $\mathbf{C V}$ & \\
\hline $\mathrm{A}$ & 62,50 & 75,00 & 68,61 & 4,80 & 7,00 & \multirow{3}{*}{$<0,001$} \\
\hline $\mathrm{B}$ & 5,66 & 40,62 & 22,91 & 11,91 & 51,98 & \\
\hline $\mathrm{C}$ & 0,00 & 14,55 & 5,46 & 7,07 & 129,50 & \\
\hline $\mathrm{D}$ & 0,00 & 31,25 & 12,34 & 13,36 & 108,32 & \multirow{5}{*}{$\begin{array}{c}\text { A }>\text { todos } \\
\mathrm{H}>(\mathrm{H}, \mathrm{E} \text { e } \mathrm{C}) \\
\mathrm{B}>(\mathrm{E} \text { e C }) \\
(\mathrm{F} \text { e } \mathrm{G})>\mathrm{C}\end{array}$} \\
\hline $\mathrm{E}$ & 0,00 & 44,26 & 10,57 & 165,17 & 152,27 & \\
\hline $\mathrm{F}$ & 0,00 & 42,49 & 20,69 & 16,90 & 81,69 & \\
\hline $\mathrm{G}$ & 0,00 & 34,72 & 16,71 & 9,24 & 55,25 & \\
\hline $\mathrm{H}$ & 0,00 & 41,66 & 27,77 & 14,60 & 52,59 & \\
\hline \multicolumn{7}{|c|}{$\begin{array}{l}\text { Nota: Grupo A } \rightarrow \text { Controle / Grupos B a H } \rightarrow \text { técnicas operatórias de autonomização } \\
\text { A probabilidade de significância refere-se ao teste } \mathrm{F} \text { da análise de variância }\end{array}$} \\
\hline
\end{tabular}

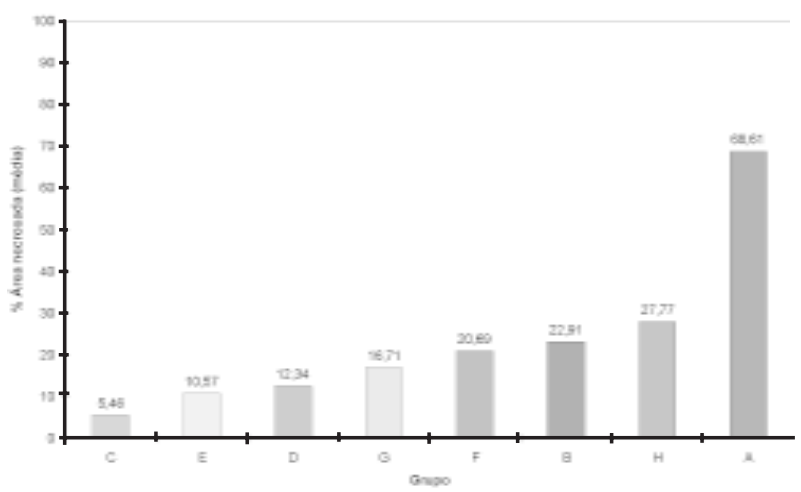

Grático 1 - Distrıbuıçāo da porcentagem de área de necrose, em média, nos grupos A, B, C, D, E, F, G e H.

\section{DISCUSSÃO}

Utilizou-se o modelo experimental de Retramcur monopediculado proposto por ÖZGENTAS et al.
(1994), com modificações em relação às medidas do retalho, que ocorreram devido a diferenças entre o peso e a espécie das ratas utilizadas nos experimentos.

Uma dificuldade notada quanto a utilização de ratas no experimento, foi a frouxidão dos tecidos e o pequeno tamanho das estruturas da parede do abdome, que exigiu cuidados técnicos no manuseio operatório e a utilização de lupa com aumento de 2,5 vezes.

A anestesia administrada associando-se o efeito analgésico do Cloridrato de Cetamina (Ketalarâ) com o efeito analgésico e relaxante muscular do Cloridrato de2-(2,6Xilidino)-dihidro-4H-tiazina (Rompunâ), mostrou-se satisfatória para a realização dos procedimentos operatórios e medidas das áreas de necrose 15.

O período escolhido para observação pósoperatória, antes do descolamento e elevação dos retalhos, após a autonomização, foi o de sete dias, sendo 
responsável por alterações estatisticamente significativas na redução da área de necrose, segundo estudo anterior de RESTIFO et al.(1997).

A medida das áreas de necrose dos retalhos foram realizadas no quinto dia pós-operatório em todos os grupos. Após a definição das áreas de necrose, observou-se início da cicatrização e contração das feridas, com progressiva eliminação das crostas de necrose.

A avaliação da influência das técnicas operatórias de autonomização no porcentual de área de necrose foi obtida pela análise de variância, cujo objetivo foi comparar os grupos em relação à variável em estudo.

Quando a análise indicou a existência de alguma diferença entre os grupos, realizou-se as comparações múltiplas de médias segundo o teste para diferenças significativas mínimas ou LSD (Least Significante Difference), utilizado quando se comparam dois ou mais grupos.

Os valores das áreas de necrose do Retramcur apresentaram variações, quando comparados a outros trabalhos da literatura, que ocorreram devido a diferenças verificadas nos métodos utilizados para obtenção das referidas medidas, o peso e a espécie dos animais estudados 10,11,12,13.

A autonomização utilizada nos animais do grupo $\mathrm{H}$, obtida por meio de incisão nos limites do retalho, associada ao descolamento da área 2 e a cauterização da perfurante musculocutânea proveniente do músculo reto do abdome direito, foi a menos efetiva na redução da área de necrose do Retramcur, ao contrário dos achados da literatura14.

O método proposto para a realização das medidas das áreas de necrose dos retalhos, mostrou-se satisfatório, possibilitando a obtenção das referidas medidas com precisão em todos os retalhos, nos quais ocorreram.

A autonomização utilizada nos animais do grupo C, obtida por meio da secção de ambos os músculos retos do abdome e cauterização de ambos os vasos epigástricos superiores profundos, mostrou-se a mais efetiva na redução da necrose do retalho musculocutâneo transverso do músculo reto do abdome monopediculado.

\section{CONCLUSÃO}

A técnica operatória de autonomização mais efetiva na redução da área de necrose do Retramcur é a que seccionou ambos os músculos retos do abdome e cauterizou ambos os vasos epigástricos superiores profundos.

\section{REFERÊNCIAS}

1. Hartrampf CR, Sheflan M., Black PW. Breast reconstruction with a transverse abdominal island flap. Plast Recontr Surg 1982;69(2):216-25.

2. Gandolfo EA. Breast reconstruction with a lower abdominal myocutaneous flap. Br J Plast Surg 1982;25:452-7.

3. Shrotria S, Websterd JT, Mansel RE, Hughes LE Complications of rectus abdominis myocutaneous flap in breast surgery. Eur J Surg Oncol 1993;19:80-3.

4. Kroll SS, Netscher DT Complications of TRAM flap breast reconstruction in obese patients. Plast Reconstr Surg 1989;84(6):886-92.

5. Wallace AM, Evans GRD, Goldberg DP, Baldwin BJ Unexpected vascular compromise in transverse rectus abdominis musculocutaneous (TRAM) flap reconstruction: a report of two pateints. Ann Plast Surg 1996;36(3):246-50.

6. Hastrampf, CRJr. The transverse abdominal island flap for breast reconstruction: a 7-year experience. Clin Plast Surg 1988;15(4):703-16.

7. Codner, MA, Bostwick JIII, Nahai F, Bried JT, Eaves, FF TRAM flap vascular delay for high-risk breast reconstruction. Plast Reconstr Surg 1995;96(7):1615-22.

8. Milton SH Experimental studies on island flaps II: ischemia and delay. Plast Reconstr Surg 1972;49(4):444-7.

9. Reinich J, Myers MB Effect of local anesthetics and epinephrine on survival of experimental flaps. Plast Reconstr Surg 1974;54(3):324-7.

10. Dorion D, Boyd JB, Pang CY Augmentation of transmidline skin perfusion and viability in transverse rectus abdominis myocutâneous (TRAM) flaps in the pig. Plast Reconstr Surg 1991;88(4):642-9.

11. Özgentas HE, Shenaq S, Spira M Study of the delay phenomenon in the rat TRAM flap model. Plast Reconstr Surg 1994;94(7):1018-24.

12. Hallock GG, Rice DC Evidence for the eficacy of TRAM flap delay in rat model. Plast Reconstr Surg 1995;96(6):1351-7.

13. Restifo RJ, Ahmed SS, Isemberg SJ, Thomson JG Timing, magnitude, and utility of surgical delay in the TRAM Flap: I. Animal studies. Plast Reconstr Surg 1997;99(5):1211-6.

14. Restifo JR, Ahmed SS, Rosser J, Zahir K; Zink J, Lalikos JA, et al. TRAM flap perforatior ligation and delay phenomenon: development of an endoscopic / laparoscopic delay procedure. Plast Reconstr Surg 1998;101(6):1503-11.

15. Massone F. Anestesiologia veterinária: farmacologia e técnicas. In: Massone F. Técnicas anestésicas em animais de laboratório. 3ed Rio de Janeiro: Guanabara Koogan; 1999. p 97-102. 
Lopes Filho R, Silva AL. Standardize of delay operations in rectus abdominis transverse miocutaneous flap: experimental study in rats. Acta Cir Bras [serial online] 2002 Jul-Aug;17(4). Available from URL: http://www.scielo.br/acb.

ABSTRACT - Objective: Standardized of delay operations and established which of delay operations is the best in reduce necrosis of the rectus transverse miocutaneous flap. Methods: 80 female Wistar rats ( 250 and 300 grams ) were divided in eight groups with ten rats $(n=10)$. The anesthesia was obteined with Ketamine hydrochloride and 2-(2,6-xilidine)5,6-dihydro-4H-1,3 thiazine hydrochloride. In the group A (control) the rectus transverse miocutaneous flap was undermined and elevated with suture in bed. In the groups B, C, D, E, F e G were accomplished differents techniques of delay operations. The measure of necrosis was accomplished in five day after operation with mould of transparency and pen of fine point in milimetral paper. Results: Statistical analysis was performed with Least Significante Difference test. In the group A (control) the percentage of necrosis was estatistical significante higher than groups B, C, D, E, F, G e H. In the group C the percentage of necrosis was estatistical significante lower than groups $\mathrm{H}, \mathrm{B}, \mathrm{F}$ e $\mathrm{G}$ and similar than groups D e E. Conclusions: The delay operation with section rectus abdominis muscles and cauterize deep epigastric superior vessels is the most efective in reduction of necrosis in the rectus transverse miocutaneous flap.

KEY WORDS - Delay. flap. Surgical flaps. Necrosis. Rectus abdominis. Rats.

Conflito de interesse: nenhum

Endereço para correspondência:

Fonte de financiamento: nenhuma

Dr. Rui Lopes Filho

R. Cônego Rocha Franco, 133/302

30430-000 Belo Horizonte - MG

Data do recebimento: 05/04/2002

Data da revisão: 18/05/2002

Data da aprovação: 02/06/2002

\title{
Visibilidade e Acessibilidade
}

da Acta Cirúrgica Brasileira

\author{
Indexada no \\ LILACS \\ INTERNET/WEB/URL: http://www.scielo.br/acb
}

Experimental version, available to search only in

Brazil, Chile and Public

www.scielo.org

Health.

Indicada pela CAPES: www.periódicos.capes.gov.br

Acesso também: www.freemedicaljournals.com

Journals by country of publication

Journals by subject

- Brazil

> Public Health

$\checkmark$ Chile

$\checkmark$ Cuba

About the project

Developing iniciatives

- SciELOModel

$>$ Costa Rica

- SciELOMethodology

sciELO Criteria

Documentation of SciELO DTD version 3.1

Guide for the installation of SciELO sites

Venezuela

SciELO Bibliography

> Related links 